\title{
EL ARQUETIPO COMO HERRAMIENTA PARA IDENTIFICAR VALORES FORMALES EN LA ARQUITEGTURA MODERNA EGUATORIANA: ARQUITEGTURA AGADÉMICA EN LA OBRA DE MARIO ARIAS SALAZAR
}

\author{
THE ARCHETYPE AS A TOOL TO IDENTIFY FORMAL \\ VALUES IN MODERN EGUADORIAN ARGHITEGTURE: \\ ACADEMIC ARGHITEGTURE IN THE WORK OF MARIO \\ ARIAS SALAZAR
}

(c) (9)

\author{
Gabriel Alejandro Moyano Tobar \\ Investigador independiente \\ Ecuador
}

Arquitecto por la Universidad de Cuenca, Ecuador, Magíster en Proyectos Arquitectónicos por la Facultad de Arquitectura y Urbanismo de la Universidad de Cuenca. Investigador independiente y propietario del estudio de arquitectura MRM Arquitectos e Ingenieros, con más de 10 años de experiencia en diseño y construcción de obras arquitectónicas de nivel público y privado en: vivienda unifamiliar y multifamiliar, edificios administrativos y de servicios, diseños urbanos de parques y plazoletas, así como en planes de ordenamiento territorial. Co-propietario de CITRAMSIT, empresa a cargo de la elaboración de estudios de movilidad, seguridad vial e investigación de tráfico.

gabrielm198@hotmail.com

orcid.org/0000-0002-1438-432X

Fecha de recepción: 06 de marzo, 2020. Aceptación: 20 de mayo, 2020. 


\section{Resumen}

En el clasicismo, la manera de hacer arquitectura seguía parámetros establecidos, contraponiéndose con la modernidad, que a raíz de los nuevos materiales y a las nuevas necesidades a causa de la industrialización, buscaba nuevas maneras de organizar forma. De esta manera se generaron cambios en los criterios formales, dando como resultado que la arquitectura moderna deje de lado la utilización de reglas fijas tal como ocurrió en el clasicismo. Términos como orden arquitectónico, tipo clasicista, modelo, entre otros, han sido empleados a lo largo de la historia para analizar una obra arquitectónica y han contribuido a determinar criterios para su análisis y estudio; sin embargo, deberían estar exentos en la investigación de edificios modernos en general, ya que la modernidad, al tener nuevos esquemas que daban respuesta a nuevas necesidades, no podía encajarse en el concepto de tipología (Piñon, 2007). El tipo en el clasicismo era una manera de clasificar los edificios por la función. En la modernidad la clasificación de los tipos desde la función no es correcta, en su lugar, procuraba una clasificación de los edificios por la forma (estructura de orden interna del edificio).

Para poder llegar a identificar valores formales en la Arquitectura Moderna ecuatoriana, es necesario entender que estos valores deben aspirar a ser universales, con criterios de forma y basados en la experiencia. En este sentido, una herramienta en este proceso podría ser la noción del arquetipo, noción que debe ser validada en la construcción de la forma, siendo la forma el resultado de relacionar el lugar, el programa arquitectónico y la construcción tal como sugiere (Gastón Guirao \& Rovira Llobera, 2007).

Entendiendo al arquetipo como un episodio de forma o la forma del tipo más allá de su función y construcción, su finalidad no es pretender reconocer el orden interno de un proyecto en particular, sino identificar valores comparativos en la construcción de forma en varias obras, diferenciando claramente que se deben reconocer relaciones, mas no identificar rasgos estilísticos e impositivos. Una vez comprendida esta noción, esta investigación se centrará en dar herramientas para reconocer e identificar arquetipos o episodios de forma en la Arquitectura Moderna a través del análisis de los edificios académicos realizados por el arquitecto quiteño Mario Arias Salazar.

\section{Palabras clave}

Arquetipo, valores formales, arquitectura. 


\section{Abstract}

In classicism, the way of making architecture followed established parameters, in contrast to modernity, which, as a result of new materials and new needs due to industrialization, sought new ways of organizing form. In this way, changes were generated in the formal criteria, resulting in modern architecture leaving aside the use of fixed rules as occurred in classicism. Terms such as architectural order, classicist type, model, among others, have been used throughout history to analyze an architectural work and have contributed to determining criteria for its analysis and study; however, they should be exempted in the investigation of modern buildings in general, since modernity, having new schemes that responded to new needs, could not fit into the concept of typology (Piñon, 2007). The type in classicism was a way of classifying buildings by function. In modern times, the classification of types from the function is not correct, instead, it sought classification of buildings by form (internal structure of the building).

In order to identify formal values in Ecuadorian Modern Architecture, it is necessary to understand that these values should aspire to be universal, with criteria of form and based on experience. In this sense, a tool in this process could be the notion of the archetype, a notion that must be validated in the construction of the form, the form being the result of relating the place, the architectural program, and the construction as suggested (Gastón Guirao \& Rovira Llobera, 2007).

Understanding the archetype as an episode of form or the form of the type beyond its function and construction, its purpose is not to pretend to recognize the internal order of a particular project, but to identify comparative values in the construction of form in various works, differentiating clearly that relationships must be recognized, but not identifying stylistic and imposing features. Once this notion is understood, this research will focus on providing tools to recognize and identify archetypes or episodes of form in Modern Architecture through the analysis of academic buildings carried out by Quito's architect Mario Arias Salazar.

\section{Keywords}

Archetype, formal values, architecture. 


\section{Introducción}

Algunos términos como tipo, tipología, modelo, entre otros, han sido empleados a lo largo de la historia para analizar y proyectar una obra arquitectónica, así como también ha contribuido a determinar criterios para su análisis, estudio y aplicación. Estos términos, que han sido estudiados por Quatremère de Quincy, Panckoucke, Durand, Alan Colquhoun, Rafael Moneo entre otros, y cuya utilización se dio en el clasicismo, permitieron determinar una serie de criterios que a lo largo del tiempo fueron evolucionando.

Quatremère de Quincy en el Dictionnaire d'architecture ${ }^{1}$ establece una diferencia entre el tipo y el modelo, concluyendo que "el tipo es una abstracción, mientras que el modelo es un objeto concreto que se copia exactamente" (De Quincy, 1832, p. 629). De manera que el modelo es una palabra que no debería aplicarse en sentido estricto en arquitectura. Por otro lado, Panckoucke explica en su Encyclopedie Methodique, Architecture ${ }^{2}$ que el copiar no es lo mismo que duplicar, de manera que la copia siempre implicará una interpretación, llegando a un nuevo objeto distinto del original; sin embargo, en la acción de duplicar no existe ningún acto intelectual (Panckoucke, 1788). Por su parte Jean Nicolás Durand en sus textos: Recueil et Parallèle des édifices de tout genre, anciens et modernes y Précis des leçons d'architecture données à l'École

Figura 1. Esquemas geométricos de Durand

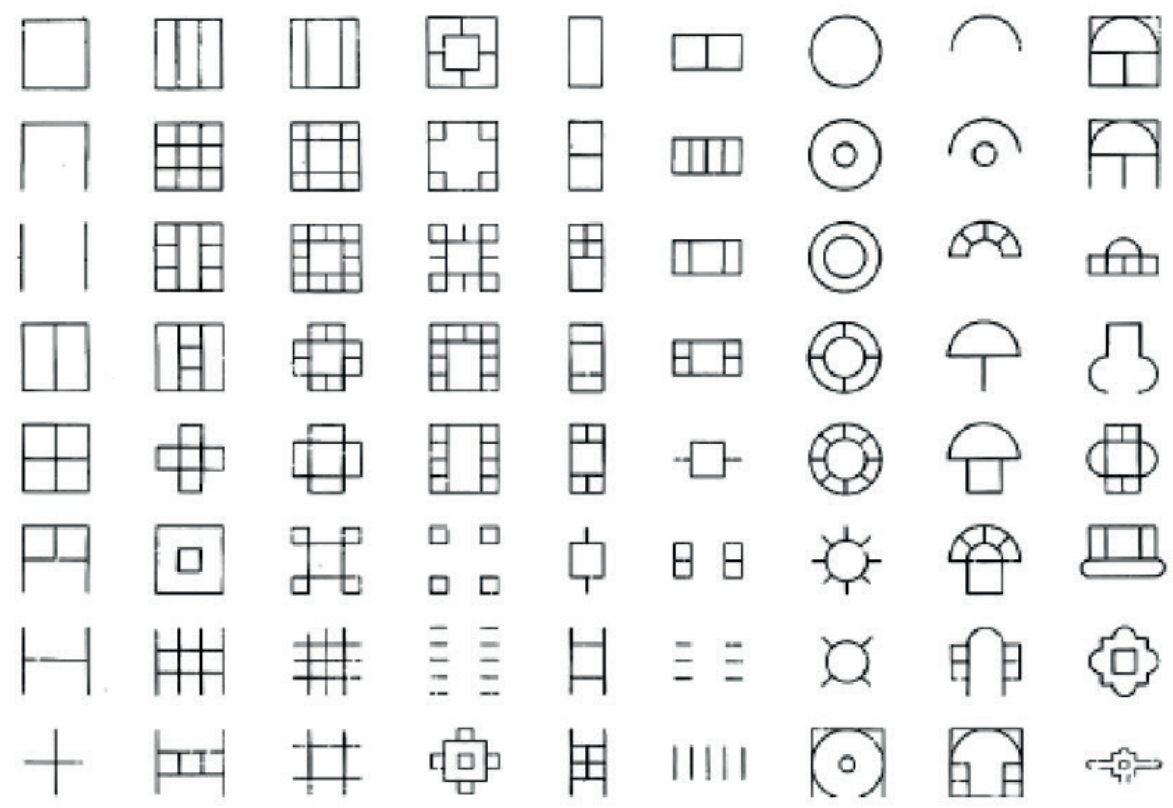

De Moyano Tobar, 2019 (Arquitectura Moderna en Edificios Académicos, p.73).

"Título de libro traducido "Diccionario de Arquitectura"

${ }^{2}$ Título de libro traducido "Enciclopedia, metodología y arquitectura" 
polytechnique ${ }^{3}$, realiza una recopilación y clasificación de edificios históricos utilizando criterios funcionales y de estilo. Durand realiza la clasificación de los edificios abstrayendo y dejando de lado el detalle, llegando a simplificar geométricamente a la arquitectura, generando así esquemas basados en la simetría, modulación, ejes y mallas, permitiendo realizar diversas maneras de organización a partir del mismo esquema compositivo, llevándolo a tener un catálogo guía al momento de empezar a proyectar (Figura 1 y figura 2).

Figura 2. Esquemas de elementos de Durand
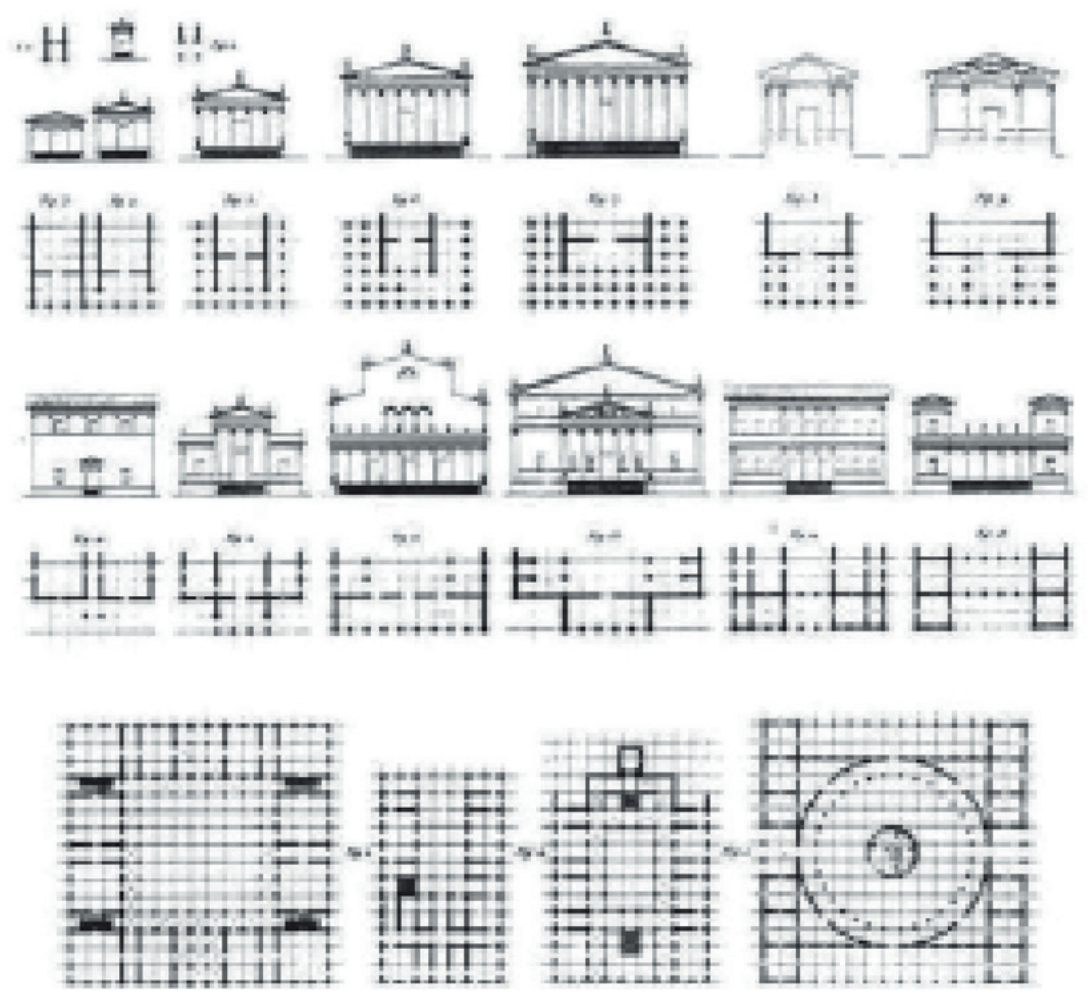

De Moyano Tobar, 2019 (Arquitectura Moderna en Edificios Académicos, p.74).

En el año 1973 el arquitecto, historiador y crítico Alan Colquhoun (1973) en su artículo "Typology as method design" afirmaba que es necesario utilizar una especie de "modelo tipológico", ya que la creación es un proceso de adaptación de formas. Por otro lado, Rafael Moneo (1978) se planteaba la siguiente pregunta ¿tiene sentido hablar hoy del concepto de tipo? Puede que se haya puesto de manifiesto que no tiene sentido el aplicar las viejas definiciones a las nuevas situaciones y que, por tanto, el concepto de tipo debe ser olvidado. Pero entender qué significa

${ }^{3}$ Título de libro traducido "Edificios antiguos paralelos de todo tipo, antiguos y modernos y Lecciones de arquitectura precisas impartidas en la Escuela Politécnica" 
el concepto de tipo es, en todo caso y hoy también, entender cuál sea la naturaleza de la obra de arquitectura (p.41).

Con esto Moneo explica que el tipo clasicista desde la función no es correcto, sin embargo, el tipo usado tan solo como una herramienta de clasificación de edificios no tiene por qué dejar de ser vigente en la arquitectura. Helio Piñón por su parte, afirma que el tipo es la característica común de los edificios, que, a lo largo del clasicismo, se utilizó mediante la clasificación tipológica, basándose en las características organizativas, constructivas y según su uso (Piñón, 2007).

También se ha utilizado la noción de tipo para intentar analizar las obras de arquitectos que marcaron la historia tal como lo hace Carlos Marcos (2012), quien analiza la arquitectura de Mies van der Rohe, deduciendo que el tipo es un espacio cuyas limitantes son una cubierta y una estructura perimetral, con la piel de cristal, que envuelve el espacio y lo protege; de esta manera categoriza sus obras en edificios bajos y altos con estructura metálica. Respecto a la relación del tipo con la arquitectura moderna, Helio Piñón (2007) comenta que "la arquitectura moderna renuncia a la autoridad de la tipología clásica por dos motivos: al no tener numerosos esquemas que dieran respuesta a nuevos programas surgidos por la industrialización y debido a los cambios en los criterios de forma." (p.138); de esta manera la modernidad no encajaría en el tipo desde un punto de vista clasicista. Piñón (2010) también comenta que "el recurso al tipo es inevitable para cualquiera que tenga sentido común" (p.2 ). Es por ello que arquitectos como Mies van der Rohe, Gordon Bunschaft 0 Mario Roberto Álvarez no han dudado en insistir en edificios arquetípicos sin llegar a repetir un modelo. Por eso, Piñón se plantea una interrogante: "¿Para qué cambiar el diseño de un edificio, si el programa es idéntico y las condiciones urbanas, técnicas y económicas, son similares?" (Reches, Diarte, y Piñón, 2010).

Si bien es cierto el término arquetipo nos puede llevar a pensar que es un retroceso al tipo cla- sicista ya que desde su significado se expresa como: "Ejemplar original o primer molde de una cosa, que reúne los elementos esenciales de un tipo o clase y sobre el que se basan las posteriores modificaciones. También llamado prototipo" (Parro, 2020).

En respuesta a lo antes mencionado, esta investigación toma como punto de partida una de las teorías del arquitecto español Helio Piñón (2012), expresado en su conferencia denominada Tipo Clasicista y Arquetipo Formal, en la cual explica que para comprender a la Arquitectura Moderna, debemos analizar la obra arquitectónica, para así lograr comprenderla sin buscar en el proyecto a los rasgos figurativos, sino encontrar los aspectos universales que se relacionen entre sí y puedan ser avalados por la historia; así la noción de arquetipo sería una manera de afrontar un proyecto, el mismo que estaría avalado por la práctica y no por la norma como en el clasicismo.

Por tanto, la finalidad de esta investigación no es el reconocer el orden interno de un proyecto en particular, sino identificar los valores comparativos de construcción de forma en varias obras, tomando al arquetipo como ejemplar arquitectónico aplicable en la Modernidad.

\section{Materiales / métodos}

La metodología desarrollada para esta investigación se centra en la utilización de imágenes y esquemas gráficos, que permitan entender las relaciones entre lugar, programa y construcción, y su incidencia en la forma del edificio.

Según Cristina Gastón y Teresa Rovira en su texto "Proyecto moderno pautas de investigación" sugieren que la manera de reconocer la arquitectura del edificio "es ponerse en el lugar del autor de la obra que se quiera conocer y volver a proyectar el edificio" (Gastón Guirao \& Rovira Llobera, 2007, p.34 ); de manera que para el análisis de la obra se debería empezar por las relaciones geográficas del terreno y su emplazamiento (lugar), luego de esto entender la solución arquitectónica de distribución y relación con el sitio (programa), y por último, la iden- 
tificación de los componentes básicos del proyecto en cuanto a lo constructivo (construcción). Con estos recursos antes indicados podemos entender todas las relaciones que nos permiten reconocer y entender la forma del edificio.

Una vez comprendida la noción del arquetipo, se intenta proporcionar herramientas para reconocer e identificar episodios de forma en la Arquitectura Moderna entendiendo al episodio "como una determinada solución arquitectónica, que responde a las condiciones de un programa y lugar, resuelto con acierto" (Saltos, 2008, p.15), para ello se ha tomado como ejemplo el análisis de los edificios académicos realizados por el arquitecto quiteño Mario Arias Salazar, quien fue parte de la primera generación de profesionales arquitectos ecuatorianos graduados en el país; siendo considerado como uno de los pioneros de la arquitectura moderna en el Ecuador. Arias fue el autor de uno de los edificios considerados como un importante referente del Movimiento Moderno en el país, la Residencia Univer- sitaria (1959), ubicada en la Universidad Central del Ecuador (UCE). Esta obra perteneció a los edificios emblemáticos que constaban en el Master Plan de Quito, cuya finalidad era el de "cambiar la cara" de la capital para así poder recibir la XI Conferencia Interamericana de Cancilleres que debía efectuarse en 1959. Arias además tuvo la oportunidad de diseñar y construir varios edificios de carácter académico dentro de la Universidad Central del Ecuador, participando en el diseño de la Facultad de Economía (1957-1959), la Residencia Estudiantil (1959) junto a Gilberto Gatto Sobral, Facultad de Agronomía (1959) y Facultad de Filosofía (1959). Dentro de la Escuela Politécnica Nacional diseñó y construyó los edificios de Ciencias Nucleares (1973), Ingeniería Mecánica (1975 y 1984), Ingeniería Civil (1975), Formación Básica (1975), Abastecimientos (1975), Ingeniería Química (1978). Este arquitecto quiteño permitió evidenciar una postura clara al momento de diseñar edificios de carácter académico (Figura 3).

Figura 3. Edificios académicos del Arq. Mario Aria Salazar en la UCE y EPN
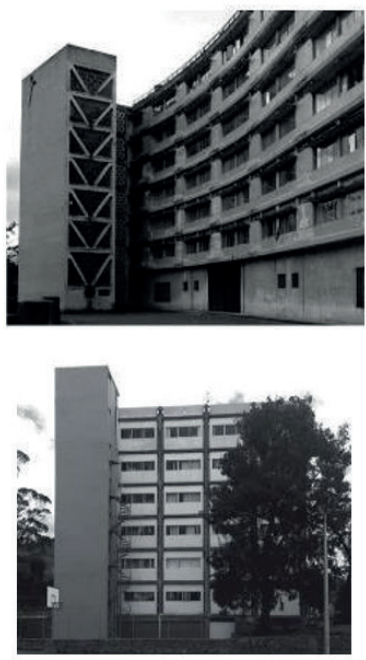
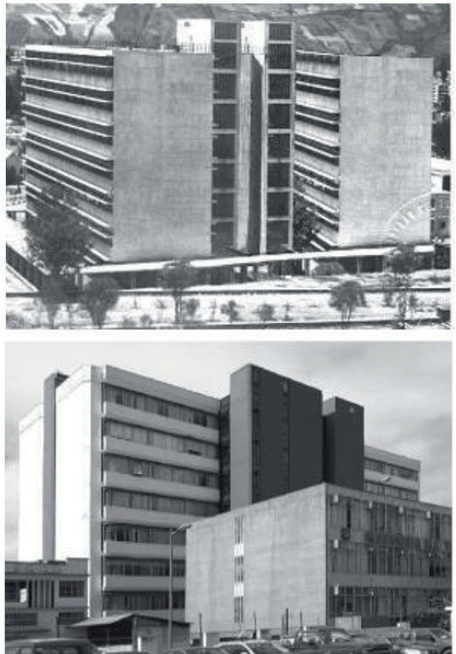
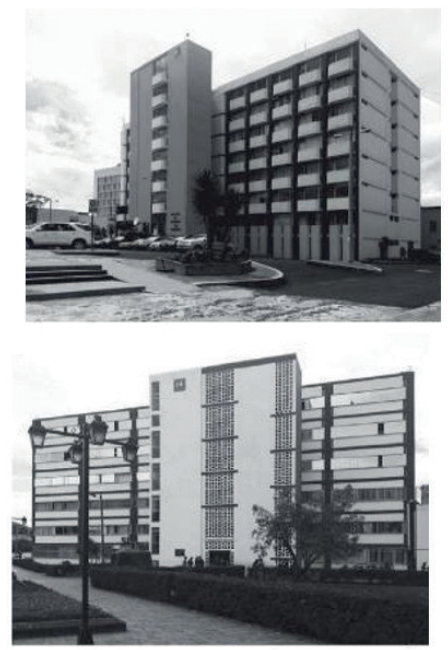

De Moyano Tobar, 2019 (Arquitectura Moderna en Edificios Académicos, pp. 60-64). 
Para abordar el análisis de los edificios y así poder llegar al reconocimiento de la forma debemos "mirar con intención", (Piñón, 2007) de manera que las mejores herramientas para poder lograrlo son la visión y la intuición, las cuales nos acercan al reconocimiento de un episodio de forma. Al utilizar estas herramientas podemos darnos cuenta que existe una manera de afrontar los edificios de carácter académico, pero para validar al episodio formal se debe comprobar su aplicación universal mediante el reconocimiento de la historia; pudiendo así constatar que varios arquitectos utilizaron este episodio aplicándolo a múltiples programas, ubicaciones geográficas e incluso sistemas constructivos diferentes.
El episodio de forma detectado, corresponde a un episodio arquitectónico que se evidencia gracias a la relación entre un volumen principal que comprende el programa y un volumen secundario que comprende las circulaciones verticales; de manera que el entender este episodio y su aplicación lo hace único en su forma de articular estos volúmenes (Figura 4).

Figura 4. Arquetipo detectado Volumen Principal - Volumen Secundario
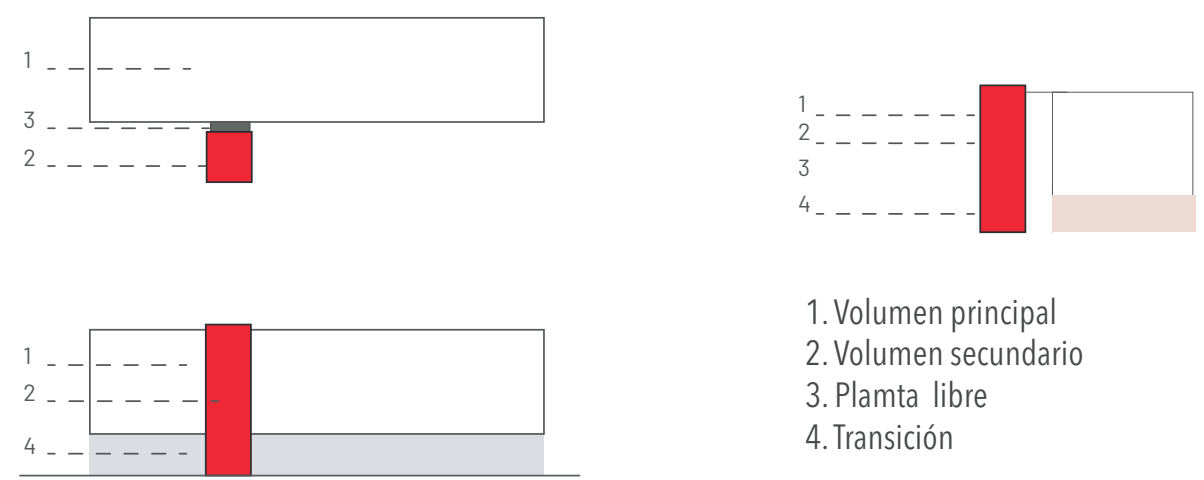

1. Volumen principal

2. Volumen secundario

3. Plamta libre

4. Transición

De Moyano Tobar, 2019 (Arquitectura Moderna en Edificios Académicos, pp. 60-64).

Para poder validar el episodio mediante la historia, fue necesario realizar una selección de edificios de diversos autores y partes del mundo, que permita entender las posibilidades y variaciones de la relación entre sus volúmenes, de manera que con ello poder rastrear un probable origen del episodio, posiblemente evidenciándolo en el Pabellón Suizo (1933) de Le Corbusier, en donde se aplica esta relación de manera coherente, teniendo un sistema en el cual el volumen que contiene el programa se encuentra libre de circulaciones verticales, teniendo una circulación horizontal para distribuir el programa; de este modo el volumen secundario se encarga de agrupar las circulaciones verticales e instalaciones (Figura 5). Por otra parte, Le Corbusier abandona la manera de articular volúmenes en sus siguientes proyectos, aplicando en la Unidad de Habitación de Marsella (1952) como algo netamente escultórico. 
Figura 5. Pabellón Suizo de Le Corbusier (1933) / reconocimiento del arquetipo
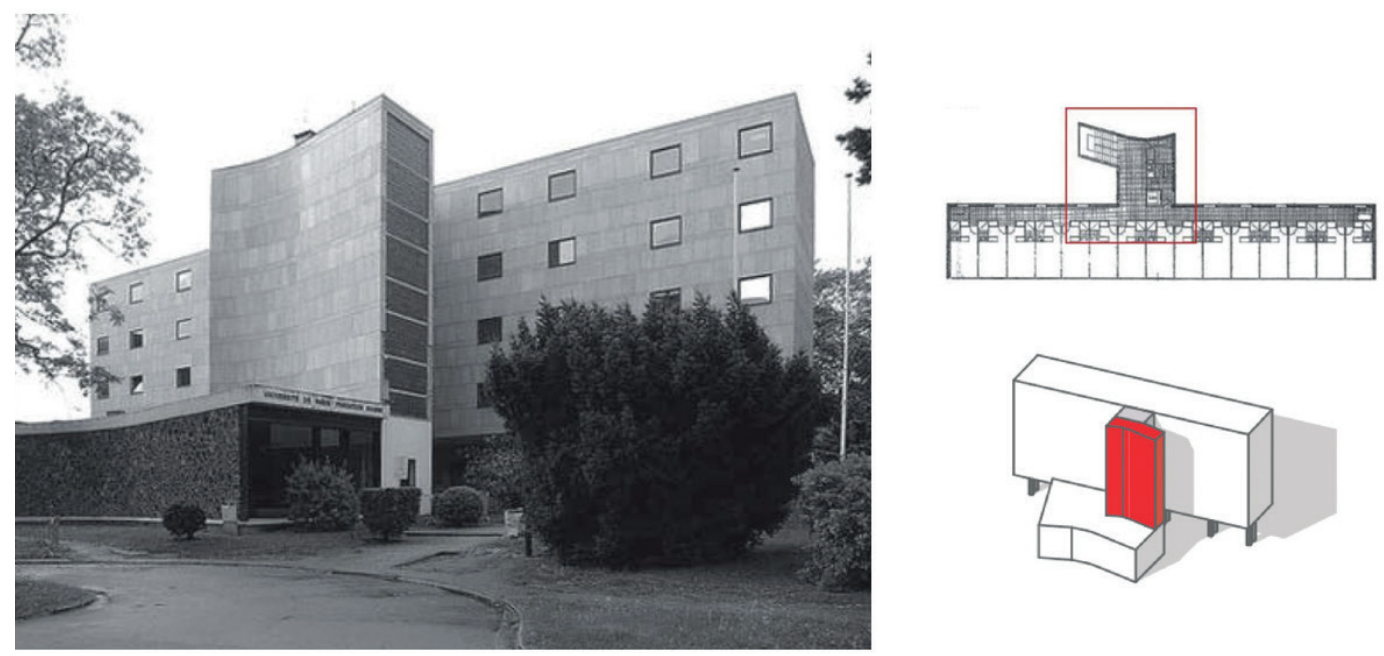

De Moyano Tobar, 2019 (Arquitectura Moderna en Edificios Académicos, pp. 87-92).

Si bien es cierto que muchos arquitectos pudieron utilizar esta manera de relacionar 0 articular los volúmenes, debemos analizar si lo realizaron aplicando al episodio de una manera figurativa, tal como lo hizo Oscar Niemeyer en sus proyectos: Palacio de Agricultura (1952), Interbau Berlín (1957) (Figura 6), Hospital da Lagoa (1958), Palace Hotel (1958), entre muchos otros. Niemeyer emplea esta forma de relacionar los volúmenes posiblemente por la influencia marcada de Le Corbusier, de manera que pudo utilizar como referente al Pabellón Suizo al momento de proponer sus edificios. Sin embargo, debido a la magnitud de los edificios no se podían cumplir las demandas de circulación que requería el programa, por lo que se adicionaron escaleras y circulaciones internas, rompiendo la relación pura entre los volúmenes. Niemeyer con estos ejemplos impone la forma al edificio, sin permitir que la forma sea un resultado de la composición y relación de sus partes (Figura 6). 
Figura 6. Edificio Interbau Berlín (1957) del Arq., Oscar Niemeyer
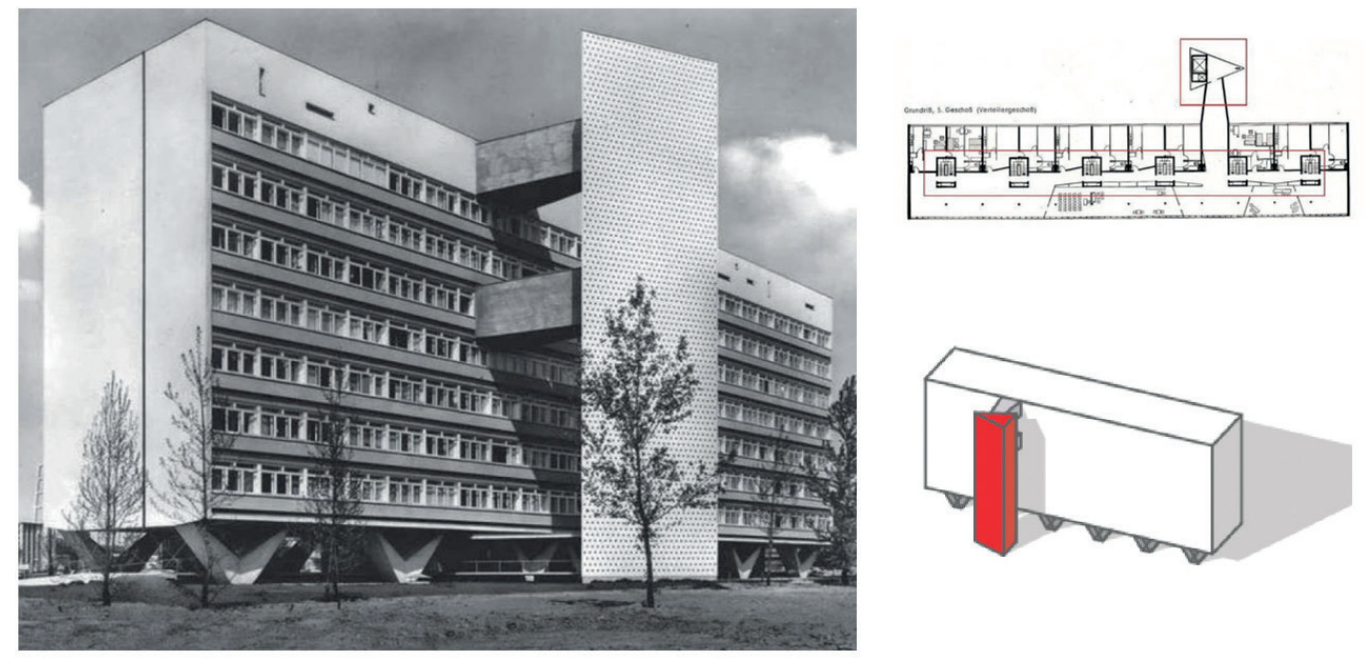

De Moyano Tobar, 2019 (Arquitectura Moderna en Edificios Académicos, pp.115-119).

Por el contrario, en el periodo que Gordon Bunshaft fue socio de la oficina estadounidense SOM (Skidmore Qwings \& Merrill), se pudo apreciar en al menos dos ejemplos claros la utilización de este recurso formal, en el edificio Inland Steel Company (1958), y en el Crown Zellerbac (1959) (Figura 7), en estos edificios la aplicación de este episodio de forma, se emplea entendiendo que al separar las circulaciones e instalaciones les permitiría dejar la planta libre sin tener obstáculos de columnas ni núcleos de circulaciones, además gracias a sus pisos técnicos se podían distribuir los espacios según las necesidades de cada uno. De esta manera, el sentido del episodio cumple las relaciones entre lugar, programa y construcción con diferente escala, materiales, etc. Bunshaft entendió que si bien el mismo recurso fue utilizado en estos dos edificios, no se llegó a repetir el objeto, cumpliendo la finalidad del episodio analizado. 
Figura 7. Crown Zellerbac (1959) del Arq. Gordon Bunshaft (SOM)
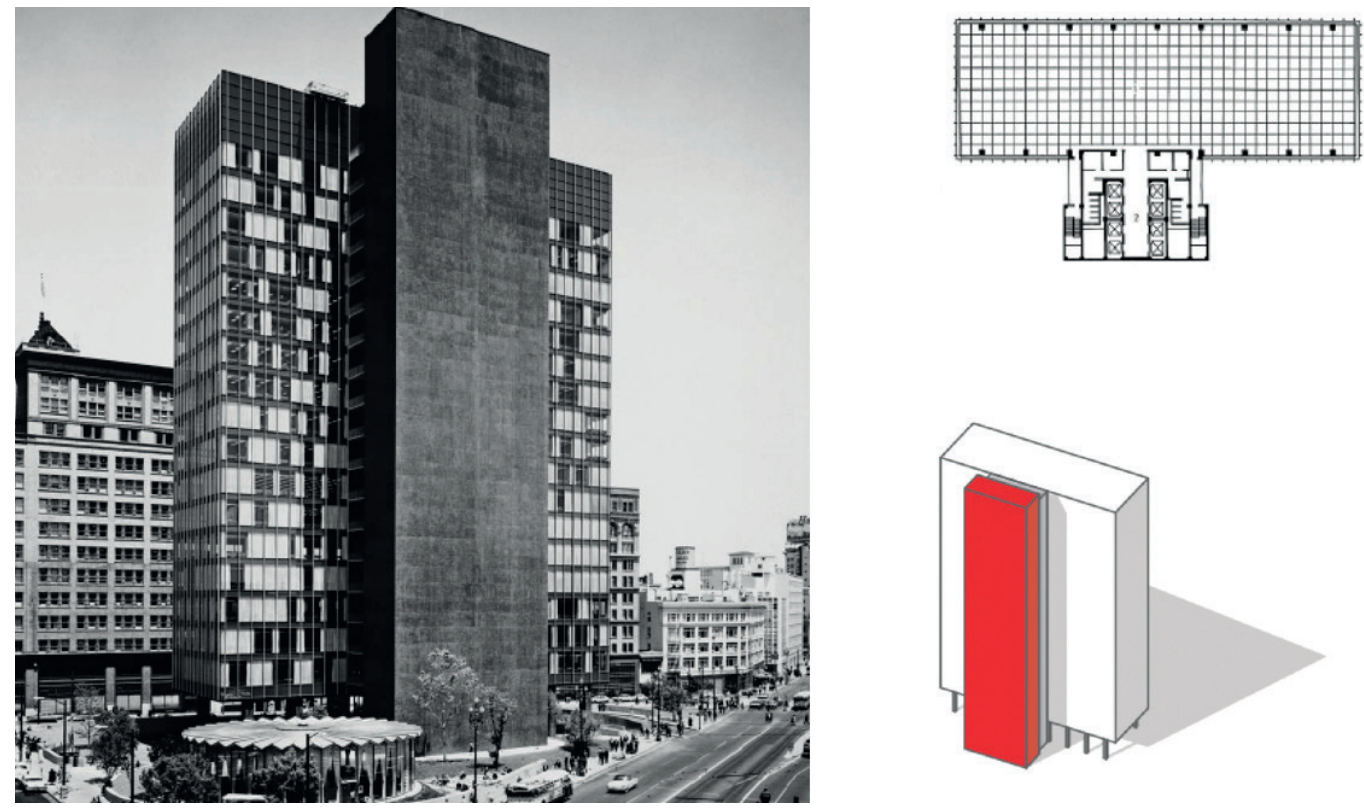

De Moyano Tobar, 2019 (Arquitectura Moderna en Edificios Académicos, pp.155- 159).

Una vez que se ha analizado el uso del episodio formal mediante proyectos de varios arquitectos con diferentes programas, métodos constructivos y en diferentes puntos geográficos (validación histórica), podemos considerar a los ejemplos locales que pudieron utilizar este recurso; tomando a uno de los edificios del arquitecto quiteño Mario Arias Salazar: la Facultad de Ingeniería Civil de la Escuela Politécnica Nacional (EPN), obra realizada en el año 1975. En este edificio es reconocible la utilización del episodio al tener un volumen que contiene el programa y otro volumen que contenga las circulaciones verticales. Esta configuración de cierta manera ya había sido utilizada en el diseño de la Residencia Estudiantil UCE (1959) y en la Facultad de Filosofía UCE (1959); de manera que el tener dos volúmenes articulados, contribuye tanto organizativamente como formalmente para su estructura formal. Arias persiste en utilizar este recurso en otros proyectos sin caer en una manera de repetir por repetir, o en una imposición de la forma, por el contrario, el haber aplicado esta postura y comprobar que funcionó (validación - experiencia), hace que insista en utilizar este episodio al momento de diseñar edificios de carácter académico.

El edificio de Ingeniería Civil de la EPN, nos ayuda de manera eficaz a poder entender este tipo de relaciones, además del papel importante que juega el agrupar las circulaciones en un volumen único. Este edificio posee ocho niveles y debido a la necesidad de un conector vertical, el arquitecto entiende que la mejor opción es agrupar y exteriorizar este volumen, dejando libre cada nivel para colocar los diferentes programas. Cada nivel contiene las aulas distribuidas gracias a un pasillo transversal, ubicándolas hacia las caras frontales y posteriores, dejando sus caras laterales ciegas todo esto debido a su orientación solar y su relación con el contexto (programa - respuesta al lugar) (Figura 8 y figura 9). 
Figura 8. Edificio Interbau Berlín (1957) del Arq., Oscar Niemeyer
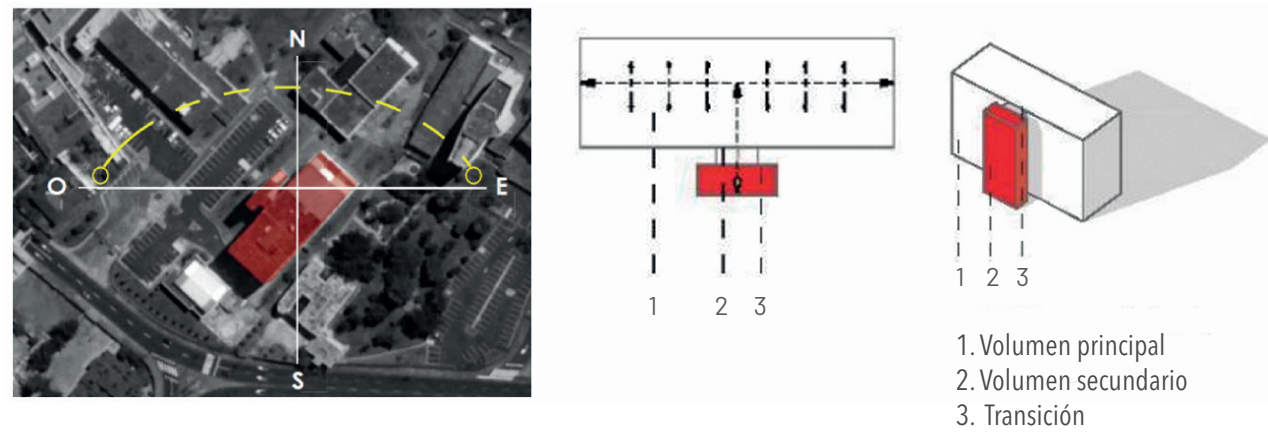

De Moyano Tobar, 2019 (Arquitectura Moderna en Edificios Académicos, pp. 294-411).

Figura 9. Edificio de la Facultad de Ingeniería Civil EPN (1957) del Arq. Mario Arias S.
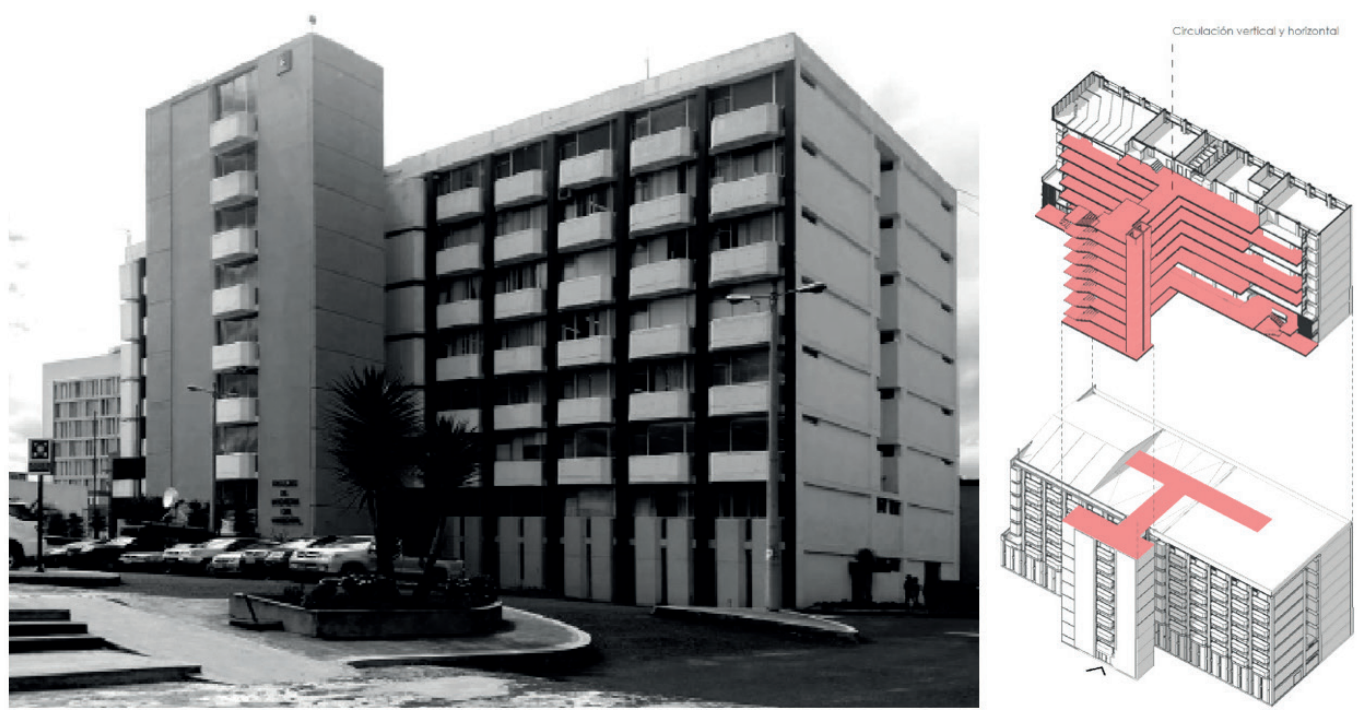

De Moyano Tobar, 2019 (Arquitectura Moderna en Edificios Académicos, pp.291- 305). 
Figura 10. Planta tipo Facultad de Ingeniería Civil EPN (1957) del Arq. Mario Arias S.

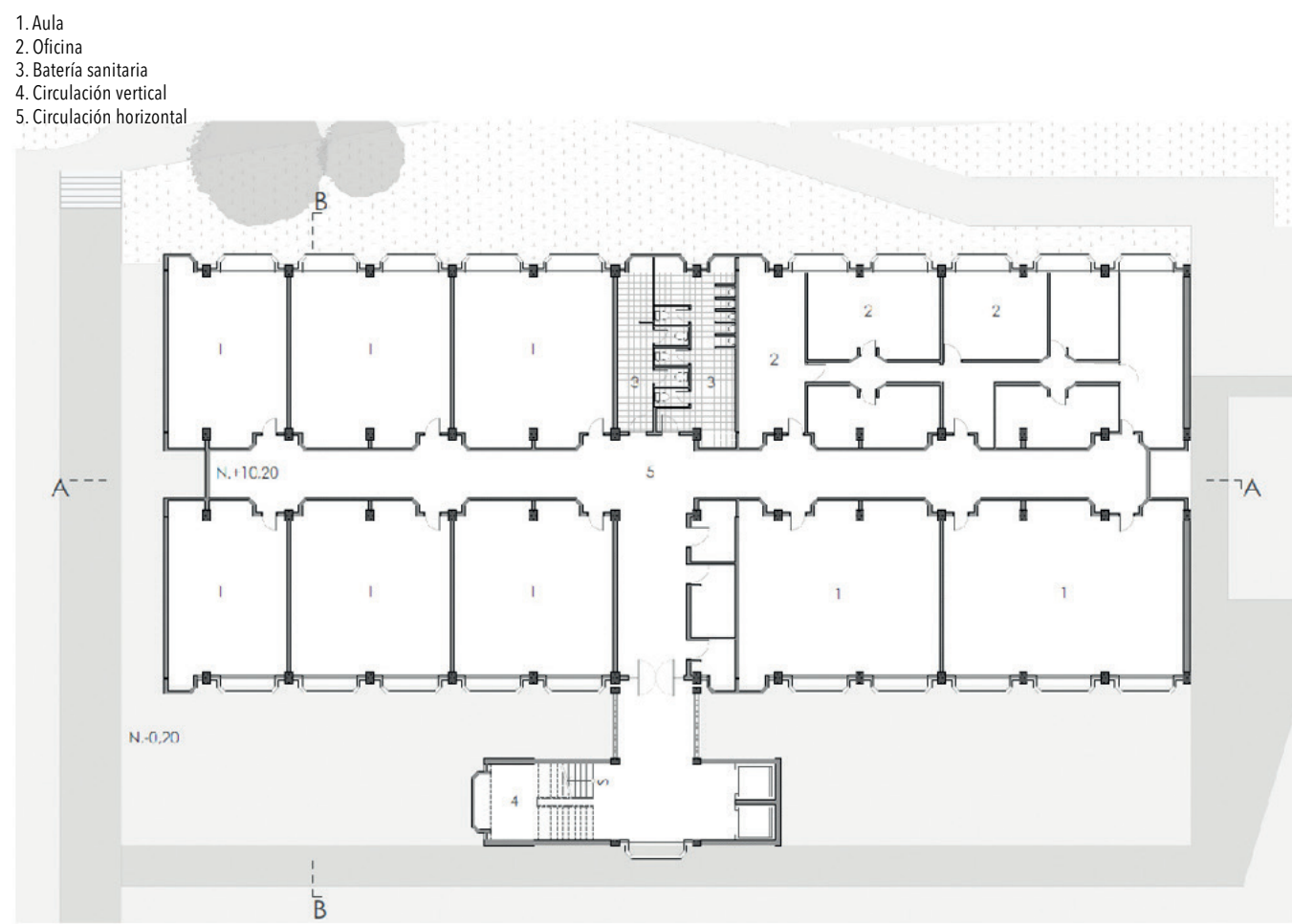

De Moyano Tobar, 2019 (Arquitectura Moderna en Edificios Académicos, p.320).

La configuración del volumen secundario no es al azar, su ubicación fue pensada de manera que los usuarios puedan tener el mismo recorrido de un extremo a otro; Esto se logra gracias a la rigurosa manera de aplicar la estructura del edificio, permitiendo organizar adecuadamente su programa (construcción - respuesta al programa) (Figura 10).
Además, para poder relacionar estos dos volúmenes (volumen principal y volumen secundario), se utiliza un recurso ya comprobado en otros proyectos, generando una transición mediante un pasillo, y utilizando además elementos tamizadores de luz que aportan a la relación visual del vínculo (Figura 11). 
Figura 11. Edificio de la Facultad de Ingeniería Civil EPN (1957) del Arq. Mario Arias S.
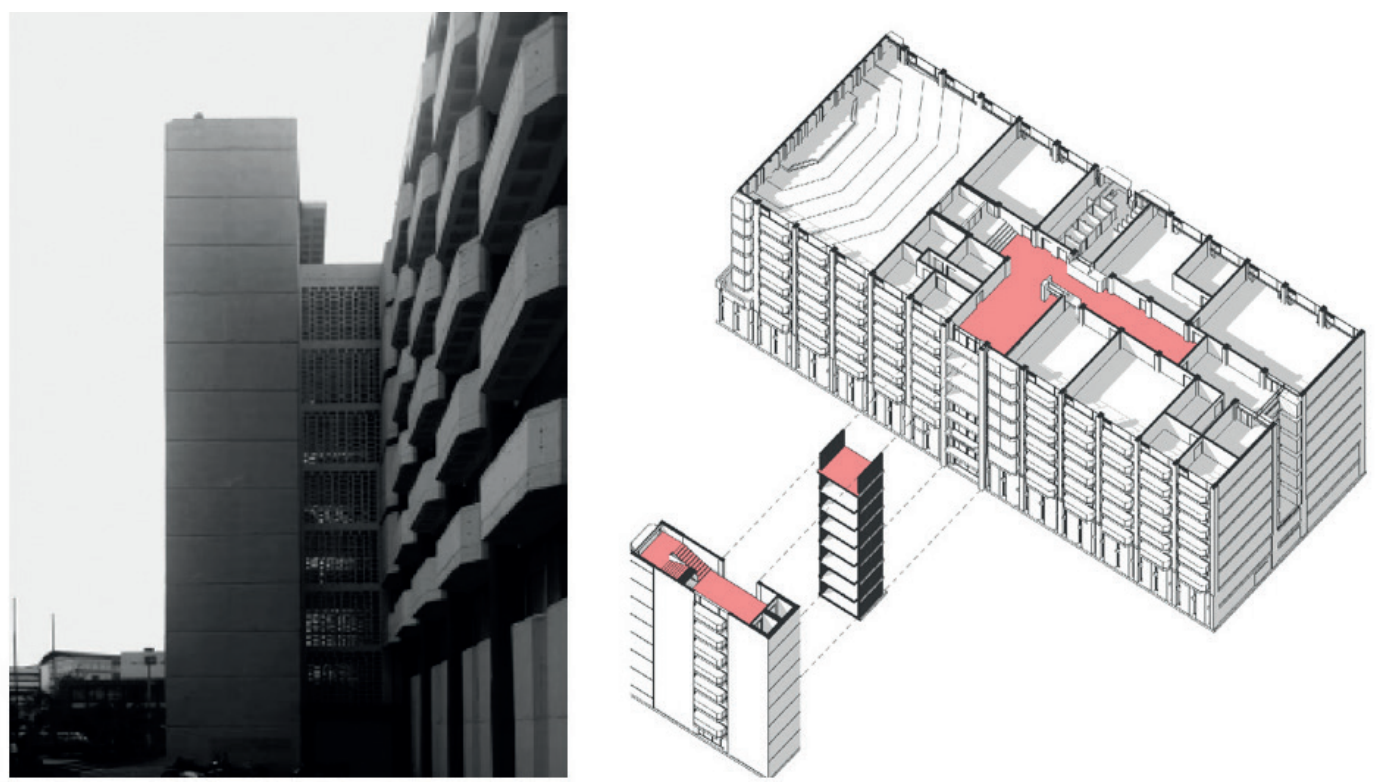

De Moyano Tobar, 2019 (Arquitectura Moderna en Edificios Académicos, pp.306- 307).

En este edificio y en otros más, como la Residencia Estudiantil (1959), Facultad de Filosofía (1959); Ingeniería Civil (1975), Formación Básica (1975), Abastecimientos (1975), Ingeniería Química (1978), Arias utiliza este episodio llevándolo a tener clara una postura definida cuando de afrontar un proyecto de edificios académicos se trata, ya que tiene preconcebido por donde debe arrancar, pero sin llegar a repetir ni duplicar ninguno de ellos, prevaleciendo su relación entre sus volúmenes. 
Figura 12. Edificio de la Facultad de Ingeniería Civil EPN (1957) del Arq. Mario Arias S.

Ingeniería Civil (1975).

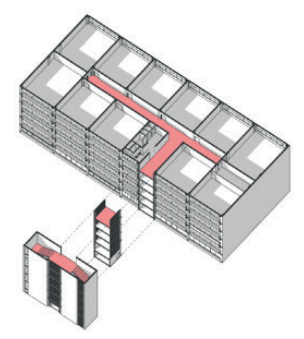

Formación Básica (1975)

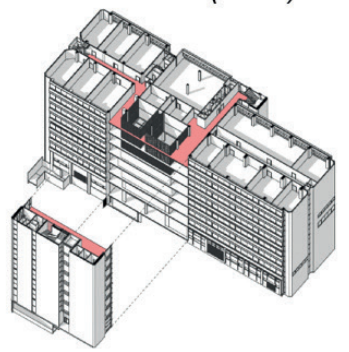

Química (1978).

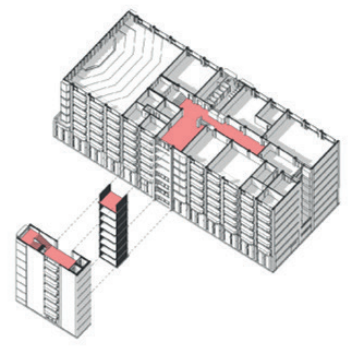

De Moyano Tobar, 2019 (Arquitectura Moderna en Edificios Académicos, pp. 307- 351- 387).

Así, sin llegar a querer descubrir el orden interno del proyecto, el episodio nos ayuda a entender el edificio, identificando las relaciones fundamentales aplicadas, relaciones funcionales, constructivas y de respuesta al lugar, de manera que este ejemplo, así como otros edificios que diseñó, nos sirve para validar la utilización del episodio al momento de iniciar un diseño.

Mario Arias Salazar pudo reconocer la relación del episodio volumen principal - volumen secundario, de manera que aplicó un sistema coherente, en donde esta relación aportó a la forma del edificio, sin abandonar su postura, teniendo en ciertos casos mejoras o retrocesos, lo que le permitió obtener múltiples resultados, sin llegar a duplicarlos, aportando a la forma del edificio. En este sentido podemos concluir que la utilización de este episodio se podría considerar como arquetípica de alguna manera acercándose a la forma en que Le Corbusier 0 Gordon Bunshaft abordaban el proyecto, al entender la relación de los volúmenes, así como la coherencia de su estructura formal.
Los criterios de diseño aplicados a nuestro medio que tiene como principal aporte el reconocimiento de un episodio de forma, es decir su arquetipo (Volumen Principal - Volumen secundario); respecto al programa si bien es cierto que cada encargo tiene el denominador común, el ser edificios de carácter académico, no se llegó a repetir un modelo, además que cada uno de ellos guarda relación con el lugar de emplazamiento dentro de los campus universitarios; su aporte en cuanto a lo constructivo, tal como se pudo observar en los referentes demuestran que la utilización de varios métodos constructivos (hormigón o acero), no afecta en sus relaciones de forma.

En el ámbito de la valoración patrimonial de la Arquitectura Moderna, la noción de arquetipo no sólo puede ser utilizada como herramienta de diseño, sino también como un instrumento que contribuya al reconocimiento o identificación de valores de un edificio. 
En este sentido, es importante comprender que para poder considerar a un episodio de forma como arquetípico, se debe utilizar en primer lugar la visión e intuición, para precisamente encontrar estos episodios de forma y ponerlos a prueba a través de la validación histórica así como de la validación por experiencia; de tal manera que se puedan determinar arquetipos en la obra de los arquitectos referentes del movimiento moderno en el país y por ende, en otras obras de arquitectura moderna del Ecuador.

\section{Referencias}

Colquhoun, Alan. "Tipología y método de diseño. "Cuadernos de arquitectura y urbanismo [en línea], 1973, Núm. 96, pp.51-53. https://www.raco.cat/index.php/CuadernosArquitecturaUrbanismo/article/view/111638/160975

Diccionario de Arquitectura y Construcción. (30 de 09 de 2019). Diccionario de Arquitectura y Construcción. http://www.parro.com.ar/definicion-de-arquetipo

Gastón Guirao, C., \& Rovira Llobera, T. (2007). El Proyecto moderno. Pautas de investigación. Universitat Politecnica de Catalunya.

Madrazo, L., 1994. Durand and the Science of Archi-tecture, Journal of Architectural Education, Vol.48, No.1, Sept., pp.12-24.

Marcos, C. L. (2012). Tipologías o Topologías. Revista de expresión gráfica arquitectónica, 17(19), pp. $102-113$

Moneo, R. (1978). On Typology. Oppositions, 22-45.

Quatremère de Quincy, A. C. (1788). Encyclopédie Méthodique. Architecture (Vol. tomo1). París.

Moyano, G. A. (2019). Arquitectura Moderna en Edificios Académicos. Aproximación a los proyectos de Mario Arias Salazar entre los años 1957 - 1978. Universidad de Cuenca.

Panckoucke, C. J. (1788). A.C. Quatremère de Quincy (Vol. tomo1). París: Encyclopédie Méthodique. Piñón, H. (2007). Sobre tipos de edificios. Quaderns d'arquitectura i urbanisme, (256), pp. 136-141.

Reches, M., Diarte, J., \& Piñón, H. (11 de Septiembre de 2010). Entrevista ISSN 2175-6708. http:// www.vitruvius.com.br/revistas/read/entrevista/11.043/3494?page $=4$

Saltos, J. (2008). Un Episodio de Forma. Cuenca: Universidad de Cuenca, Facultad de Arquitectura, Centro de Posgrados, p. 15. 